\title{
Modelling and simulation as research tools in paediatric drug development
}

\author{
Francesco Bellanti • Oscar Della Pasqua
}

Received: 30 November 2010 / Accepted: 2 December 2010 / Published online: 19 January 2011

(C) The Author(s) 2011. This article is published with open access at Springerlink.com

\begin{abstract}
Purpose Although practical and ethical constraints impose special requirements for the evaluation of treatment safety and efficacy in children, the main issue remains the empirical basis for patient stratification and dose selection at the early stage of the development of new chemical and biological entities. The aim of this review is to highlight the advantages and limitations of modelling and simulation $(\mathrm{M} \& \mathrm{~S})$ in supporting decision making during paediatric drug development.

Methods A literature search on Pubmed's database Medical Subject Headings (MeSH) has been performed to retrieve relevant publications on the use of model-based approaches in paediatric drug development and therapeutics.

Results M\&S enable the assessment of the impact of different regimens as well as of different populations on a drug's safety and efficacy profile. It has been widely used in the last two decades to support pre-clinical and early clinical drug development. In fact, M\&S have been applied to drug development as decision tools, as study optimization tools and as data analysis tools. In particular, this approach can be used to support dose adjustment in specific subgroups of a population. M\&S may therefore allow the individualisation of drug therapy in children, improving the risk-benefit ratio in this population.
\end{abstract}

F. Bellanti $\cdot$ O. Della Pasqua $(\triangle)$

Division of Pharmacology,

Leiden/Amsterdam Center for Drug Research,

P.O. Box 9502, 2300 RA Leiden, The Netherlands

e-mail: odp72514@gsk.com

O. Della Pasqua

Clinical Pharmacology \& Discovery Medicine,

GlaxoSmithKline,

Stockley Park, UK
Conclusions The lack of consensus on how to assess the impact of developmental factors on pharmacokinetics, pharmacodynamics, efficacy and safety has so far prevented a broader use of M\&S. This problem is compounded by the limited collaboration between stakeholders, which prevents data sharing in this field. In this article, we emphasise the need for a concerted effort to promote the effective use of this technology in paediatric drug development and avoid unnecessary exposure of children to clinical trials.

Keywords PKPD modelling - Paediatric drug development . Paediatric investigational plan · Clinical pharmacology . Bridging studies $\cdot$ Clinical trial simulation

\section{Introduction}

Model-based drug development represents an invaluable resource in pharmaceutical research and development [1]. The recent introduction of regulatory requirements for the development of medicines for children will have a farreaching impact on how evidence can be generated on the risk-benefit ratio of novel treatments for paediatric diseases. These requirements make the application of model-based approaches an obligatory step in paediatric drug development. In this paper we show how modelling and simulation $(\mathrm{M} \& \mathrm{~S})$ have been applied to drug development as decision tools, as study optimisation tools and as data analysis tools. These applications are split into three main sections, with special focus on how these different domains can support drug discovery, and non-clinical and clinical development. In addition to the role of mechanistic models, which are surfacing from research in systems biology and systems pharmacology, and their contribution to the rationale for patient selection and paediatric dosing regimen, practical and ethical limitations 
imposed by empirical protocols are highlighted. The landscape is then completed with an overview of the implications of M\&S for the advancement of the concept of personalised medicines in children. Ultimately, this manuscript attempts to emphasise the need for less empirical evidence and for a more systematic, integrated evaluation of the overall risk-benefit ratio of novel treatments in children.

\section{Systems biology and systems pharmacology}

The use of computer-aided mathematical simulations to describe biological processes and systems is a fundamental part of systems biology [2]. The objective of such simulations is a model-based prediction of the behaviour and the dynamics of biological systems. In this manuscript, focus is placed on the role of modelling and simulation (M\&S) in systems pharmacology and paediatric diseases. In this context, models can be used to quantitatively characterise how drugs affect the dynamics of biological systems as well as the regulatory mechanisms triggered by a given pharmacological intervention [3].

Because of the complexity of biological systems simplified models are often used $[4,5]$. However, the quality of modelbased predictions strongly depends on the quality of the model, which in turn is defined by the quality of the data and the profoundness of the knowledge it is based on. Whilst simplified models have been particularly useful for interpreting clinical data and developing novel biomarkers, complex models may be required to predict the overall clinical response or to quantify the role of modulating individual pathways or targets in health and disease conditions.

These requirements have resulted into two different approaches for the evaluation of the dynamics of biological systems, namely a "bottom-up" and a "top-down" approach. The "bottom-up" approach, historically used by biologists, brings together all the known pieces at a subsystem level with the objective of identifying a formal structure of the whole system; a clear drawback is that it does not account for possible unknown factors. In contrast, the "top-down" approach departs from an observable and clinically relevant behaviour and then iteratively identifies the biological components, which could yield or cause such behaviour. Both methods are complementary and have a wide range of applications [6-9]. Despite the differences in the focus of each approach, over the last few years, it has become clear that to fully understand the complexity of biological organisms they must be studied as whole systems; the "top-down" approach seems to satisfy this requirement [2].

The use of M\&S in drug development has contributed to the advancement of translational research, allowing the analysis of complex biological systems and their interactions with chemical and biological entities (i.e. drugs and biologics). This field has evolved into what is currently defined as systems pharmacology. In conjunction with additional statistical concepts, M\&S has become a powerful tool for predicting drug effects across a wide range of conditions, including extrapolation from in vitro to in vivo, from animal to humans, from health to disease, from short- to long-term effects.

Despite the increase in the use of M\&S as tools for decision-making in pharmaceutical $R \& D$, their benefits as an optimisation and data analysis tool has remained undervalued and sometimes ignored by key stakeholders [10, 11]. This attitude appears contradictory to ethical and scientific tenets, which should underpin the evaluation of the risk-benefit ratio in special populations, such as children. The ethical constraints and practical limitations associated with clinical research clearly impose new alternative methodology to ensure accurate assessment of treatment response in these patients. In that sense, the value of $M \& S$ to paediatric research may be even greater than the evidence available so far for drug development in adults. The interest in M\&S is also reaching the attention of the regulatory authorities. In April 2008, the European Medicines Agency (EMA) organised a "Workshop on Modelling in Paediatric Medicines" [12]. More recently, M\&S have been proposed as a framework for the evaluation of drugs by regulators taking into account different clinical scenarios [7, 13].

\section{Clinical research in paediatric diseases}

As indicated previously, the purpose of the manuscript is to evaluate the use of M\&S as an alternative approach to the design, analysis and interpretation of experiments and clinical protocols in paediatric drug development. Despite some limitations, M\&S enable systematic, integrated evaluation of drug and disease properties, providing quantitative measures of treatment response across a wide range of clinical and statistical designs, some of which would not be feasible in real-life (i.e. due to exclusion criteria). Furthermore, M\&S can overcome many of the pitfalls associated with the use of empirical protocols and isolated, sequential developability criteria.

One of the greatest challenges in paediatric drug research is to find the appropriate dosing regimen. It should be noted that in spite of the ICH E11's explicit requirement for appropriate evaluation of medicinal products for children, today about $70 \%$ of the medicines given to the paediatric population and $93 \%$ of the medicines given to critically ill neonates remain unlicensed or used off-label [14-16]. Even if a large number of studies have been performed in paediatrics over the last few decades, the empiricism upon which clinical drug development is based often results in ineffective or unsafe treatments. To ensure that appropriate dose rationale and dosing regimens are used in paediatric trials, as well as to identify potential subgroups of patients who may be more susceptible to treatment response and/or adverse 
events, it is essential to characterise the underlying pharmacokinetic-pharmacodynamic (PKPD) relationships $[17,18]$. PK and PD properties may change in children over the whole age continuum, and these changes must be considered, especially when interpreting non-clinical safety pharmacology and toxicology data $[14,17,18]$.

Understanding the effects of medicinal products in paediatric patients is an important goal. However, this should be done without compromising the well-being of paediatric patients participating in clinical studies. This responsibility is shared by companies, regulatory authorities, health professionals and society as a whole [20]. It is clear that traditional drug development approaches do not satisfy the aforementioned requirement. In contrast, $M \& S$ can be used to address various practical, scientific and ethical issues that arise in paediatric research.

\section{Empiricism in paediatric drug development}

The majority of drugs on the market have been developed primarily for adults [21]. Several constraints have been used to justify the poor assessment of efficacy and safety in the paediatric population, and consequently provide appropriate labelling recommendations for children. These constraints can be categorised into three classes, namely: practical, ethical and regulatory.

Practical issues are principally the increasing cost of clinical development and the availability of patients required to satisfy the statistical power of each study [22] (i.e. these criteria cannot be applied to paediatric patients with rare diseases). Patient autonomy and unforeseen adverse events represent some of the ethical factors that limit the application of empirical experimental design in paediatric drug research $[15,23]$. These limitations constrain physicians to extrapolate data from the adult population and to normalise dosing regimens to a child's body weight or body surface area without evidence of linear correlations for the changes in the parameters of interest across populations (see examples in Table 1) [16, 24].

The FDA's paediatric study decision tree is very clear in recommending bridging and dose selection from adults to children, and its purpose is to streamline the costs and time required to develop drugs in the paediatric population [21]. The bridging rationale, and as such the data extrapolation, can be justified only if the following conditions are all met. Adults and children have to present:

1. The same disease progression

2. Similar PKPD relationships

3. Similar endpoints

If these requirements are not met, further PKPD or efficacy studies are needed. We anticipate that M\&S methodology can result in important improvement in the planning, implementation and analysis of such studies [7]. In fact, the ICH E11 already proposes the use of population PK analysis in paediatric studies in order to facilitate the protocol design and to reduce practical and ethical constraints [20].

From a regulatory perspective, lack of working knowledge and understanding of $M \& S$ concepts create an additional hurdle to the effective use and implementation of the approach in regulatory submissions. Despite the opportunities for the use of $\mathrm{M} \& \mathrm{~S}$ by regulatory guidelines, empiricism still plays a main role in drug development. As recently shown by our group, a keyword-based search performed on 95 European Public Assessment Reports (1995-2007) reveals that only 22 out of the 95 documents analysed refer to the use of M\&S methodologies. Furthermore, these EPARS do not include keywords, such as biosimulation, PKPD modelling or clinical trial simulation.

\section{Modelling and simulation}

In addition to the insight into the underlying pharmacological mechanisms and dynamics of a biological system, M\&S also enable the assessment of important statistical elements. The integration of these elements is currently known as pharmacometrics. In pharmacometric research, three important components are characterised, namely: a drug model, a disease/placebo model and the implementation model (trial design and decision criteria). Whilst modelling enables translation of the relevant features of a system into mathematical language (i.e. model parameters), simulation allows the assessment of a system's performance under hypothetical and real-life scenarios (i.e. "what-if" scenarios), yielding information about the implication of different experimental designs and quantitative predictions about treatment outcome, dosing requirements and covariate effects (Fig. 1) [7, 24, 25].

In this regard, the great advantage of the use of M\&S in paediatric drug development is the possibility of exploring relevant scenarios before enrolling children into a clinical protocol. Simulations allow evaluation of a range of parameter values (dose, clearance, etc.), including an assessment of critical scenarios, such as overdosing, that cannot be generated in real-life studies [15]. Most importantly, it enables systematic assessment of the impact of uncertainty.

Modelling and simulation can be used not only as a learning and decision-making tool, but also as a design optimisation and data analysis tool. Consequently, it can support the selection of candidate drugs and streamline decisions regarding first-time human, PKPD and safety/efficacy clinical studies [7, 24, 26]. Furthermore, great attention is being paid to study design before the implementation of an experiment or clinical 
Table 1 Examples of drugs commonly used in paediatric medicine for which the paediatric dose is not linearly correlated with body weight. Details on the clinical implication of non-linearity between drug exposure and descriptors of body size can be found in Cella et al. [18]

\begin{tabular}{|c|c|c|c|}
\hline Drug & $\begin{array}{l}\text { Therapeutic } \\
\text { indication }\end{array}$ & Adult dose & Paediatric dose \\
\hline Chloramphenicol & Bacterial infection & $50 \mathrm{mg} / \mathrm{kg} /$ day & $50 \mathrm{mg} / \mathrm{kg} /$ day; neonates: $25 \mathrm{mg} / \mathrm{kg} /$ day \\
\hline Carbamazepine & Epilepsy & $5-8 \mathrm{mg} / \mathrm{kg}$ every $12 \mathrm{~h}$ & $\begin{array}{l}\text { > } 12 \text { years: } 5-8 \mathrm{mg} / \mathrm{kg} \text { every } 12 \mathrm{~h} \text {; children: } 3-10 \mathrm{mg} / \mathrm{kg} \text { every } 8 \mathrm{~h} \\
\text { infants: } 3-10 \mathrm{mg} / \mathrm{kg} \text { every } 8 \mathrm{~h}\end{array}$ \\
\hline Phenytoin & Epilepsy & $2 \mathrm{mg} / \mathrm{kg}$ every $12 \mathrm{~h}$ & $\begin{array}{l}\text { Children: } 2.3-2.6 \mathrm{mg} / \mathrm{kg} \text { every } 8 \mathrm{~h} \text {; infants: } 2.3 \mathrm{mg} / \mathrm{kg} \text { every } 8 \mathrm{~h} \text {; } \\
\text { neonates: } 2.5-4.0 \mathrm{mg} / \mathrm{kg} \text { every } 12 \mathrm{~h}\end{array}$ \\
\hline Propofol & Anaesthesia & $\begin{array}{l}<55 \text { years: } 6-12 \mathrm{mg} / \mathrm{kg} / \mathrm{h} \\
>55 \text { years: } 3-6 \mathrm{mg} / \mathrm{kg} / \mathrm{h}\end{array}$ & 2 months to 16 years: $7.5-18 \mathrm{mg} / \mathrm{kg} / \mathrm{h}$ \\
\hline Busulfan & Cancer & $0.8 \mathrm{mg} / \mathrm{kg}$ every $6 \mathrm{~h}$ & $\leq 12 \mathrm{~kg}: 1.1 \mathrm{mg} / \mathrm{kg}$ every $6 \mathrm{~h} ;>12 \mathrm{~kg}: 0.8 \mathrm{mg} / \mathrm{kg}$ every $6 \mathrm{~h}$ \\
\hline Tobramycin & Bacterial infection & $3 \mathrm{mg} / \mathrm{kg} / \mathrm{day}$ & $\begin{array}{l}\text { Children: } 6 \text { to } 7.5 \mathrm{mg} / \mathrm{kg} / \text { day; }<2 \text { weeks: } 4 \mathrm{mg} / \mathrm{kg} / \text { day; with } \\
\text { cystic fibrosis: } 10 \mathrm{mg} / \mathrm{kg} / \text { day }\end{array}$ \\
\hline Enfuvirtide & HIV & $180 \mathrm{mg} /$ day & $\begin{array}{l}11-15.5 \mathrm{~kg}: 54 \mathrm{mg} / \mathrm{day} ; 15.6-20 \mathrm{~kg}: 72 \mathrm{mg} / \text { day; } 20.1-24.5 \mathrm{~kg}: \\
90 \mathrm{mg} / \mathrm{day} ; 24.6-29 \mathrm{~kg}: 108 \mathrm{mg} / \mathrm{day} ; 29.1-33.5 \mathrm{~kg}: 126 \mathrm{mg} / \text { day; } \\
33.6-38 \mathrm{~kg}: 144 \mathrm{mg} / \text { day; } 38.1-42.5 \mathrm{~kg}: 162 \mathrm{mg} / \text { day }\end{array}$ \\
\hline Oseltamivir & Influenza & $150 \mathrm{mg} /$ day & < $15 \mathrm{~kg}: 60 \mathrm{mg} / \mathrm{day} ; 15-23 \mathrm{~kg}: 90 \mathrm{mg} /$ day; $23-40 \mathrm{~kg}: 120 \mathrm{mg} /$ day \\
\hline Nelfinavir & HIV & $2.5 \mathrm{~g} /$ day & $\begin{array}{l}7.5-8.5 \mathrm{~kg}: 0.8 \mathrm{~g} / \mathrm{day} ; 8.5-10.5 \mathrm{~kg}: 1 \mathrm{~g} / \text { day; } 10.5-12 \mathrm{~kg}: 1.2 \mathrm{~g} / \mathrm{day} ; \\
12-14 \mathrm{~kg}: 1.4 \mathrm{~g} / \text { day; } 14-16 \mathrm{~kg}: 1.6 \mathrm{~g} / \text { day; } 16-18 \mathrm{~kg}: 1.8 \mathrm{~g} / \text { day; } \\
18-22 \mathrm{~kg}: 2.1 \mathrm{~g} / \text { day }\end{array}$ \\
\hline Digoxin & Heart failure & $1.4-4.0 \mu \mathrm{g} / \mathrm{kg} / \mathrm{day}$ & $\begin{array}{l}\text { Children: } 3-8 \mu \mathrm{g} / \mathrm{kg} / \mathrm{day} \text {; infants: } 7.5-12 \mu \mathrm{g} / \mathrm{kg} / \text { day; neonates: } \\
4-8 \mu \mathrm{g} / \mathrm{kg} / \mathrm{day}\end{array}$ \\
\hline
\end{tabular}

protocol. In brief, $\mathrm{M} \& \mathrm{~S}$ can be applied to the development of a new drug from the first steps in discovery to the approval stage. Later in therapeutics and clinical practice, $M \& S$ can guide dose adjustment for specific subgroups of a population and enable the evaluation of the implications of relevant factors, such as treatment adherence, changes in formulation and drug combinations [22, 27].
Like all sciences, best practices should be followed when performing M\&S. To fulfil this objective the following issues must be clearly defined a priori:

1. The objective(s) of the M\&S exercise

2. The criteria for data selection and the exclusions or limitations of the dataset

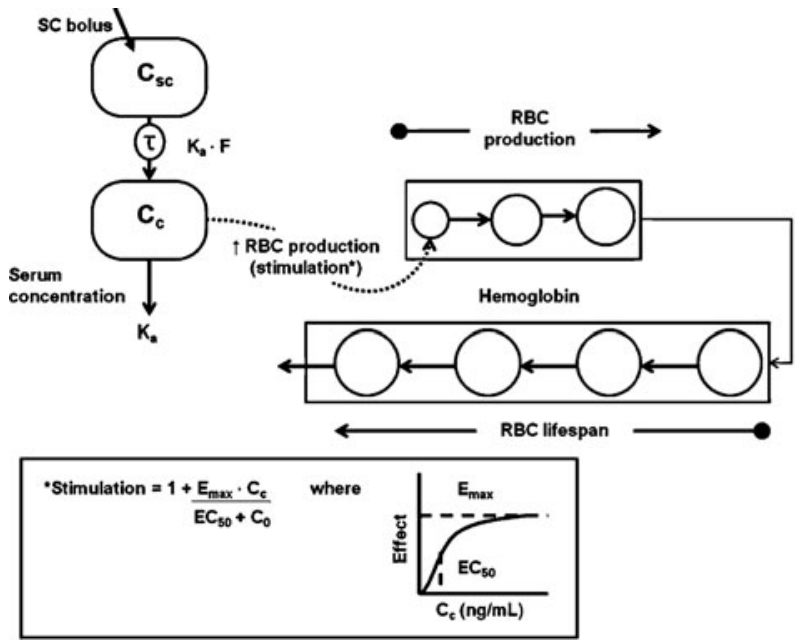

Fig. 1 Simulations allow the assessment of a system's performance under hypothetical and real-life scenarios (i.e. "what-if" scenarios), yielding information about the implications of different experimental designs and quantitative predictions about treatment outcome. In this

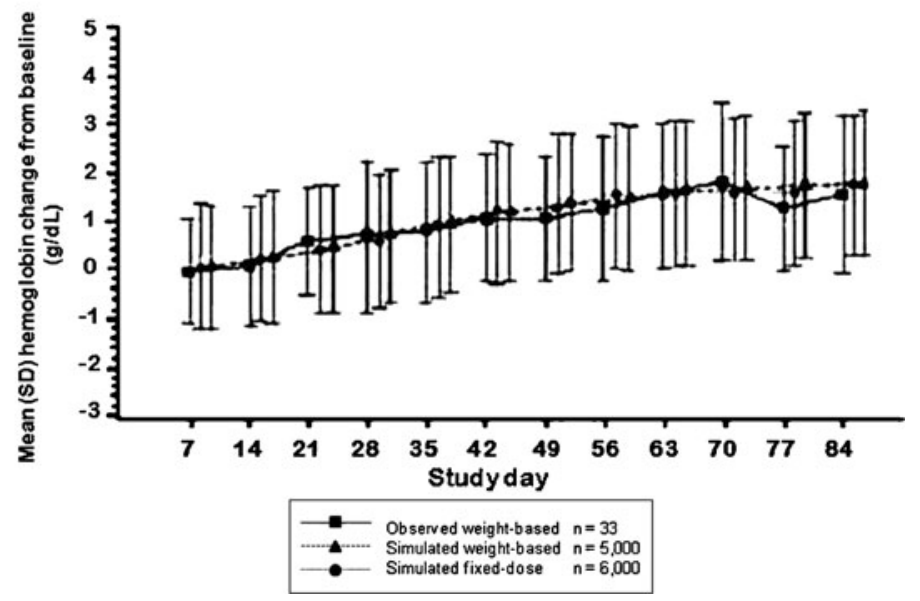

example, a model of haematopoiesis is used to simulate the effects of darbepoetin alfa administered every 2 weeks in chemotherapy-induced anaemia based on weight-based fixed-dosing regimens. Adapted from Jumbe et al. [25] 
3. Assumptions and rationale for model selection or simulation features

4. The statistical method, algorithm and methodology

5. Model qualification or validation criteria

It should be noted that the workflow and tools should have an audit trail and be validated to ensure reproducibility of the findings $[7,27]$. The advantages and drawbacks of model-based approaches from drug discovery to the clinical practice will be highlighted in the following paragraphs.

\section{M\&S in drug discovery}

During lead optimisation and candidate selection go/no-go decisions have to be made. From the very first step of development of an new molecular entity (NME), absorption, distribution, metabolism and elimination (ADME) information is required to understand the drug's properties in vivo $[28,29]$. The application of $\mathrm{M} \& \mathrm{~S}$ methodologies at this stage will support and facilitate decision-making processes. Predictive models assist the selection of appropriate candidates, as well as the design of in vivo PK studies [30]. The obvious advantage of this application is the possibility of integrating in vitro to in vivo properties as well as to pharmacodynamic characteristics, identifying differences in drug performance in vivo, as opposed to decision-making based on isolated developability criteria. This concept has been recently applied in the evaluation of COX2 inhibitors [31]. Furthermore, M\&S allow optimisation of experimental protocols.

At this stage, pharmacokinetics can also be evaluated by studying each part of the ADME process in an integrated manner. Physiologically-based pharmacokinetic (PBPK) models provide an integrated view of drug disposition in vivo [28]. In contrast to empirical compartmental models, a PBPK model is aimed at describing the in vivo behaviour of the drug before the acquisition of in vivo data. PBPK relies primarily on describing drug disposition in terms of organ distribution, blood flow and metabolic capacity (Fig. 2). This allows better understanding of PK properties, more rational candidate selection, and extrapolation of dose levels, of routes of administration, and of data across species. This approach has some appealing features in that predictions can be made about the need for changes in dosing regimen because of developmental and other agerelated factors $[7,28,30]$.

The relevance of this type of information is evident already at the lead optimisation stage: better and quicker understanding of a drug's PK profile in vivo may drastically improve the decision-making process. Nevertheless, it is worth highlighting that the predictive value of these models depends on the selection of correct model parameterisation and on the availability of suitable descriptors (i.e. experimen- tal data reflecting the appropriate set of physicochemical properties) [29].

M\&S in non-clinical drug development

At the non-clinical phase in vitro and in vivo animal studies are the main source of information about pharmacokinetic and pharmacodynamic properties. The objective at this stage is to further improve the understanding of the drug properties in vivo and to extrapolate findings, identifying correlations or making predictions about a drug's performance in humans (i.e. clinical response).

Juvenile toxicological studies, which involve young animals, have been used to investigate a drug's pharmacology and toxicology. Findings are extrapolated assuming a correlation between developmental growth in animals and children [32]. Even if the assumptions and rationale can be supported for some indications, numerous issues have to be addressed to allow appropriate interpretation of the findings. In contrast, $M \& S$ can optimise the use and interpretation of those data, enabling a mechanism-based, systematic extrapolation of the data across species (e.g. scaling exposure findings for differences in metabolic clearance). Furthermore, it allows quantitative assessment of age- or growth-related differences in drug effects and consequently the potential implications for different paediatric age groups [19, 21].

In addition, the techniques available at this stage, such as PBPK and PBPK-PD models [26], can use in vitro data to predict plasma and tissue concentrations [33]. This implies substantial reduction in the number of animals per experiment and sometimes the replacement of animals by in silico experiments. Also in this case, the use of a model-based approach allows optimisation of experimental protocols, improving the accuracy and efficiency of data extrapolation.

In summary, the benefits from $M \& S$ methodologies at the non-clinical stage include the prediction and characterisation of primary PK parameters (PBPK models) and pharmacodynamic properties (PBPK-PD models). Model parameters can then be used to predict the dose range to be tested in clinical studies, including the requirements for optimal sampling and study design [7, 26, 27].

M\&S in clinical drug development

Limited availability of patients and practical constraints, such as difficulties in blood sampling, have often been used as justification for the lack of systematic evaluation of drug response in children [14-16]. M\&S can address many of these limitations, but its wide implementation in clinical development has remained wishful thinking. This is partly due to the lack of understanding and working knowledge in quantitative pharmacology and pharmacometrics by spon- 
Fig. 2 Physiologically-based pharmacokinetic (PBPK) models provide an integrated view of drug disposition in vivo. In contrast to empirical compartmental models, a PBPK model is aimed at describing the in vivo behaviour of the drug before the acquisition of in vivo data. PBPK relies primarily on describing drug disposition in terms of organ distribution, blood flow and metabolic capacity. Actual experiments become confirmatory and can therefore be optimised in terms of dose range, sample size, frequency and sampling intervals. Diagram adapted from Theil et al [28]

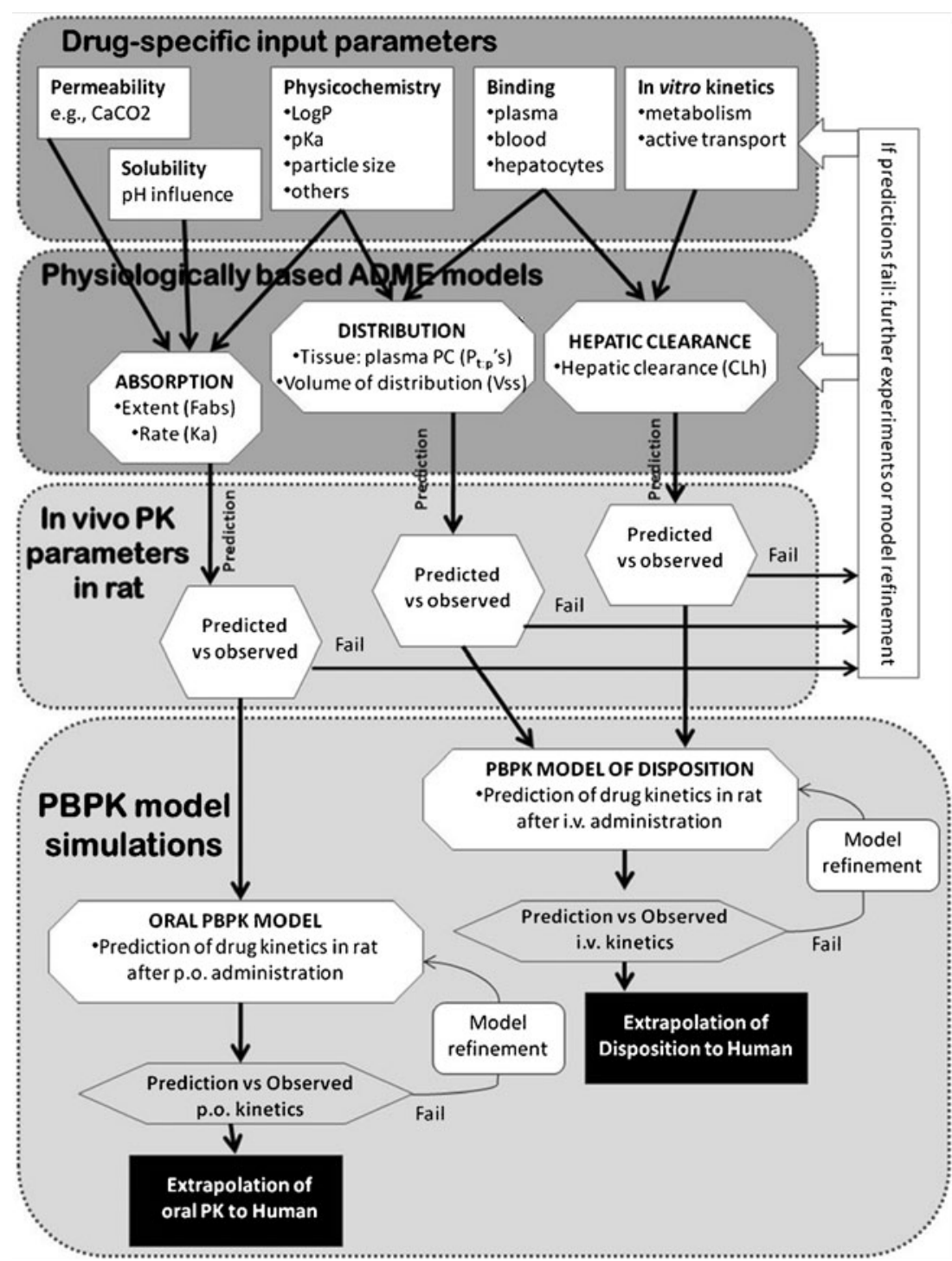

sors, regulatory agencies and investigators (e.g. paediatricians, statisticians, research nurses) who are responsible for the planning, design and/or approval of clinical trials.

\section{PBPK and disease models}

The difficulties in performing paediatric trials constrain physicians in extrapolating data from the adult population to children. For this purpose, simple allometric methods based on body weight or body surface area have been frequently used. However, particularly in neonates and infants, the use of the allometric approach may fail to identify the appropriate dosing range [16, 21]. Once more PBPK models may play a pivotal role in the estimation of dosing requirements across the paediatric population. Physiological differences between adults and children and between different age groups can be incorporated into the model to evaluate variation in pharmacokinetics. This may allow conversion of the exploratory nature of first-in children studies into a confirmatory step [21].

Application of bridging techniques requires however further understanding of disease. Therefore, disease and disease progression models need to be considered when comparing drug response and kinetics in adults and children [7]. Disease models can also be applied to simulate treatment response. In combination with drug models, it is possible to explore the implications of different algorithms for dose adjustment [7]. The use of disease models to evaluate drug-disease interactions and the role of covariates in pharmacokinetics, pharmacodynamics and treatment outcome demand the use of somewhat sophisticated statistical methods, which cannot be achieved by standard linear regression techniques. These methods often rely upon Bayesian statistical concepts and include parameterisation based on hierarchical, non-linear mixed effects models, also known as the population approach. 


\section{Population models}

Population methods consider the population rather than the individual as the object of the investigation. The approach is particularly suitable when information on individual subjects is limited (i.e. sparse sampling). In fact, this is a common situation in pharmacokinetic and pharmacodynamic studies in children. Hence, it would be already possible to circumvent the aforementioned practical and ethical issues in paediatric research [14, 34]. It is unfortunate that the expertise is still limited to allow its widespread use in drug development. Conceptually, population models rely on pooled data across treatment cohorts or even across different studies [15], which is of great importance considering that the number of paediatric patients in some diseases may be extremely limited. Moreover, one can evaluate different clinical scenarios without exposing children to any risk, and explore drug, disease or covariate effects in a larger number of virtual patients compared with what is observed in the patients enrolled in a real trial $[15,35]$. A further advantage is the possibility of assessing the clinical relevance of covariates to drug exposure and to evaluate simultaneously their effect on the treatment response [34]. As an example, Knibbe et al. recently reported a population pharmacokinetic model to describe propofol disposition in children aged 1 to 5 years. In contrast to what happens in adults, the model showed the body weight to be a covariate for clearance [36].

Population pharmacokinetic (pop PK) and pharmacokineticpharmacodynamic (pop PKPD) models basically comprise the representation of three main components: a structural model that describes pharmacokinetics or pharmacodynamic characteristics (e.g. two-compartment disposition, sigmoid Emax); a statistical model describing between-subject variability and an error model that accounts for the residual variability. Most importantly, population models incorporate the effect of influential covariates (e.g. weight, age, pharmacogenetics etc.) $[7,14,15]$ on model parameters (e.g. CL, EC50), instead of correlating them directly with the observed variables. This is particularly appealing, as it prevents the bias common to empirical methods aimed at the assessment of covariate effects in the presence of non-linear pharmacokinetics and complex PKPD relationships [27]. This concept is clearly illustrated by Ihmsen et al., who applied a PKPD model to characterise the delayed onset and prolonged recovery to rocuronium. The authors show the impact of disease on drug potency when comparing healthy subjects with patients affected by Duchenne muscular dystrophy [37].

Another concept introduced into paediatric research is the KPD model. This represents a specific group of nonlinear mixed effect models that have been developed to describe exposure-effect relationships in the absence of drug concentration measurements [7, 38]. This approach is very useful if drug elimination from the biophase is the rate-limiting step in drug disposition [38]. The approach is, however, not suitable for extrapolating data across different scenarios (e.g. different doses, or populations) for which no observations are available [7].

The availability of population PK and PKPD models offers an important opportunity as a study optimisation tool (e.g. dose selection, sampling times, treatment duration and population size). These models can also be used to support prediction and extrapolation of data across different age-groups, dosing regimens and formulations or delivery forms (Fig. 3) [7, 14, 26, 27, 39]. Moreover, population models may enable extrapolation of long-term efficacy and safety based on short-term pharmacokinetic and treatment response data.

\section{$M \& S$ and biomarkers}

A biological marker or biomarker is defined as a characteristic that is objectively measured and evaluated as an indicator of normal biological or pathogenic processes or pharmacological responses to a therapeutic intervention [31, 40]. Biomarkers can be directly measured or derived by model-based approaches and expressed as model parameters. In drug discovery and drug development a validated biomarker may facilitate decision-making, supporting the prediction of treatment response as well as guide dose adjustment. If validated accordingly for sensitivity, specificity and clinical relevance, biomarkers can also be used as surrogate endpoints (symptoms or signs that constitute one of the target outcomes). In this context, model-based analysis of biomarker data can contribute to validation procedures and enable comprehensive sensitivity analysis, with a clear understanding of the sensitivity and specificity rates (i.e. false-positive and false-negative rates). The availability of biomarkers may also be a determinant in the progression of a clinical trial when the clinical outcome is delayed or difficult to quantify in short-term studies [31, 40].

Another important advantage of model-based approaches is that they allow access to functional components and structures of a biological system that cannot be identified experimentally. The best example of such a concept is the quantification of insulin sensitivity, as defined by the insulin sensitivity index. The loss in insulin sensitivity because of diabetes progression cannot be measured direct from insulin and glucose levels in plasma; it is derived from a model. In addition, M\&S provide insight into how drug treatments may alter disease [41, 42].

\section{Clinical trial simulation}

In contrast to meta-analysis, clinical trial simulation (CTS) enables the assessment of the impact of a range of design characteristics on the statistical power to detect a treatment effect prior to exposing patients to an experimental drug. In a field where most clinical trials have a conservative design, 


\section{a}

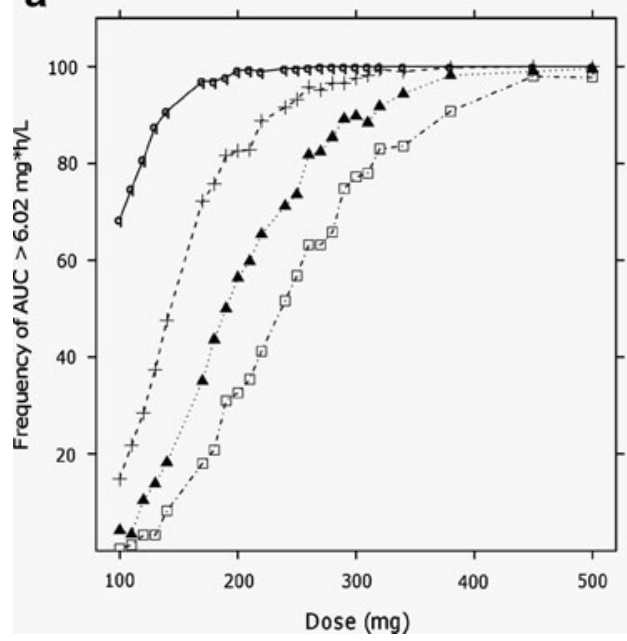

Fig. 3 Modelling and simulation can be used to support prediction and extrapolation of data in early clinical development. The graphs show the implications of pharmacokinetic differences for systemic exposure across different age groups in children. Based on pharmacokinetic parameter estimates systemic exposure can be simulated for a range of dosing regimens. Note the non-linearity in the dose

this methodology offers a unique opportunity to evaluate innovative designs. Rather than performing power calculations that only take sample size and endpoint variability into account, CTS allows calculation of power taking into account a multitude of other factors.

In general, CTS utilises two types of models [43]. First, a drug-action (PKPD) model is considered, which comprises pharmacokinetic and pharmacodynamic factors. In chronic diseases the model also accounts for disease progression. Unfortunately, the lack of knowledge about the mechanisms underlying treatment response in many therapeutic indications has prevented the development of mechanistic PKPD models. Hence, examples often refer to standard statistical models, such as e.g. the mixed model for repeated measures (MMRM). Such statistical models have however a downside

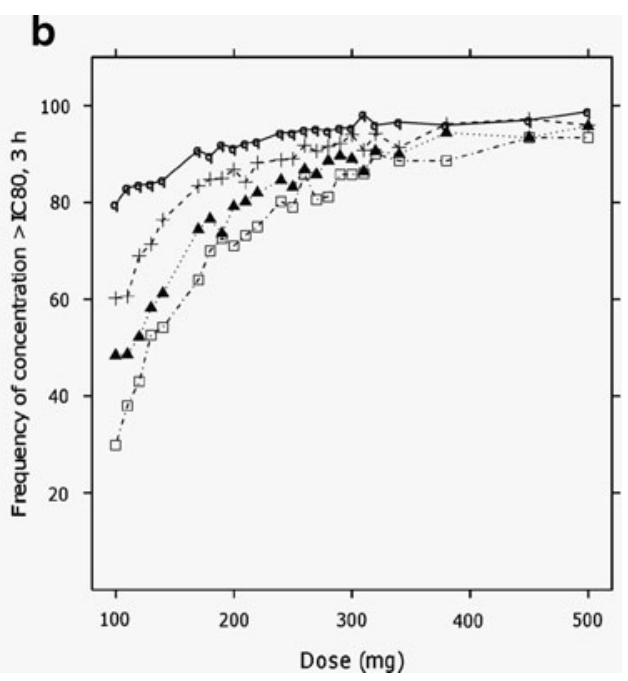

range for the different age groups. Lines depict the fraction of patients categorised by body weight reaching target exposure criteria $\left(\mathrm{a}=\mathrm{AUC}>6.02 \mathrm{mg} * \mathrm{~h} / \mathrm{L}, \mathrm{b}=\right.$ plasma concentrations above $\mathrm{IC}_{80}$ for at least $3 \mathrm{~h}$ ) following different doses of abacavir. circles $10 \mathrm{~kg}$, crosses $20 \mathrm{~kg}$, triangles $30 \mathrm{~kg}$, squares $40 \mathrm{~kg}$. From Cella et al [10]

in that they often do not incorporate concentration-effect relationships and therefore do not allow for inferences about age-related differences in pharmacokinetics, as is the case for paediatric populations. Second, CTS requires a trial execution model. These models simulate other important aspects of the trial, such as dropout, compliance and protocol deviations (Fig. 4) [44]. In this manner, one can determine all possible outcomes under candidate trial designs, allowing such trial designs to be compared in a strictly quantitative manner. Thus far, very few examples exist in which relevant design factors have been evaluated prospectively as part of the planning of a paediatric trial.

It is also important to stress that CTS allows investigation of factors that cannot be scrutinised by meta-analysis or empirical design. First, designs which have not been implemented cannot

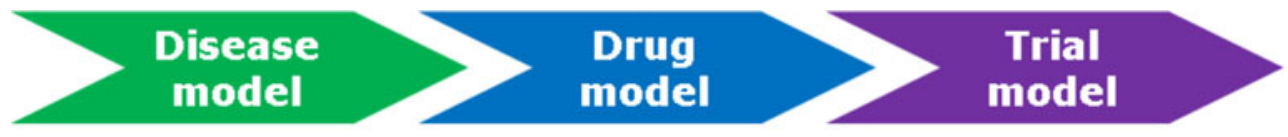

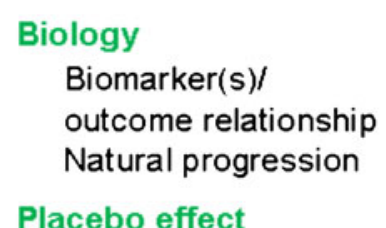

Placebo effect

\section{Pharmacology Effectiveness Safety \\ Preclinical/Healthy $/$ Patient}

\section{Product features}

Fig. 4 The diagram depicts the major components of a clinical trial simulation (CTS). In model-based drug development, a CTS can be used to characterise the interactions between drug and disease, enabling among other things the assessment of disease-modifying effects, dose selection and covariate effects (e.g. age, body weight). In conjunction with a trial model, CTS allows the evaluation of such interactions, taking into account uncertainty and trial design factors, including the implications of different statistical methods for the analysis of the data 
be included in a meta-analysis. Second, it is difficult to separate the influence of multiple design factors, whereas CTS allows evaluation of a single factor at a time. Although meta-analyses may provide valuable information about differences in patient populations and treatment response, it is unfortunate that many investigators consider overall publication review sufficient to gather evidence on the role of design factors, as often suggested in the discussion of meta-analysis results.

If simulated data is to be exchangeable with actual patient data, it is imperative that not only model parameters are unbiased, but that estimates of variability are also accurate. Often interpretation of statistical model results focuses on the predicted values of the treatment effect. This does not necessarily mean that response distributions reflect what occurs in the true patient population. In fact, it is not infrequent to see model mis-specifications being corrected by inflated estimates of variability. It is therefore critical for clinicians to understand that standard goodness-of-fit criteria do not take simulation characteristics into account and may therefore not be indicative of the best model. Such a comparison between simulated and original data can be performed using graphical and statistical tools.

CTS relies on the availability of accurate model parameter and corresponding distributions to investigate "what if" scenarios across a different range of conditions or design features, such as population size, stratification levels, dose range, sampling scheme, and even different endpoints. One of the main advantages of such a virtual or statistical experiment is the possibility to predict 'trial performance' and so to identify potential limitations in study and protocol design prior to its implementation [7, 24, 45, 46]. In fact, some clinical trial simulations have been evaluated against outcomes from real trials. They showed accuracy and an important correspondence between simulated and "real" results [27]. For instance, Nguyen et al. have developed a new dosing regimen for busulfan in infants, children and adolescents through the use of population PK model. The new regimen has been accepted and adopted as conditioning treatment prior to haematopoietic stem-cell transplantation in paediatric patients since 2005 [47]. Another example of rational drug dosage is evident in the study from Laer et al. where population PK modelling and simulations have been applied to develop age-based dosing regimens for sotalol in children with supraventricular tachycardia. For children $<6$ years the identified dose was higher than the one for neonates and children $>6$ years [48].

M\&S and personalised medicines

A CTS represents one of the most obvious methods of exploring the concept of personalised medicine and its implications in clinical practice. M\&S techniques can be applied to identify patient subgroups and tailor dosing regimen for specific subsets of the population [14, 22]. PBPK-PD models, pop PK and pop PKPD models, as well as disease models can all be used for this purpose [7, 19, 21, 27, 49]. The use of a model-based approach for personalised medicines also permits better scrutiny of diagnostic and prognostic factors, including quantitative estimates of differences in the risk-benefit ratio for a given group of patients or treatment option (Fig. 5). Despite the
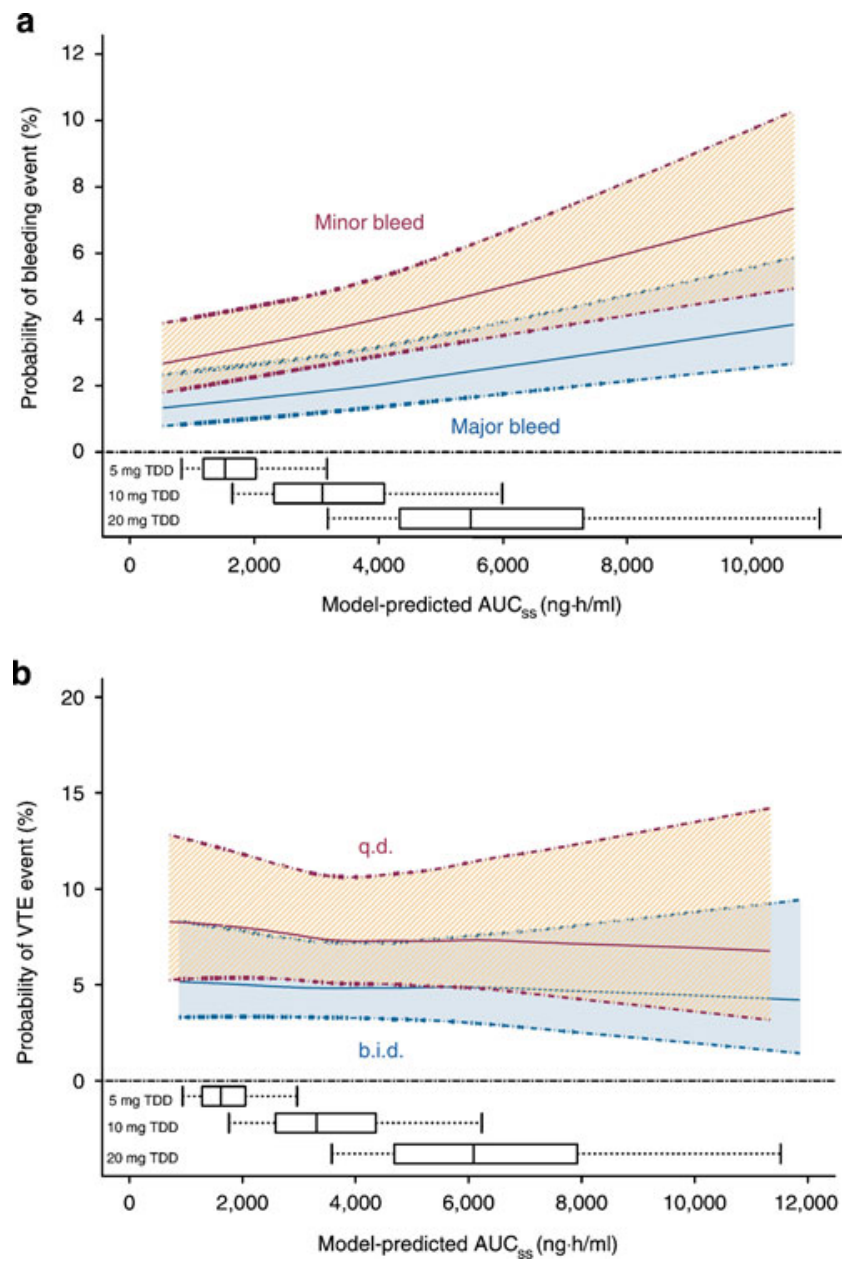

Fig. 5 The concept of personalised medicines implies quantitative assessment of the risk-benefit ratio at the individual and patient population levels. M\&S techniques are critical for such an evaluation. The use of a therapeutic utility index (TUI) illustrates how the safetyefficacy ratio of a treatment correlates with drug exposure. The graphs show a safety: probability of a major or minor bleeding event as a function of daily steady-state apixaban exposure $\left(\mathrm{AUC}_{\mathrm{ss}}\right)$; $\mathbf{b}$ efficacy: probability of a venous thromboembolic event (VTE) as a function of daily apixaban $\mathrm{AUC}_{\mathrm{ss}}$ and regimen. The shaded regions surrounding the regression lines represent the $90 \%$ bootstrap confidence intervals. The boxes at the bottom of each figure represent the distribution of apixaban exposures for the doses indicated. Exposure distributions are shown for the total daily dose (TDD) because the distributions of $\mathrm{AUC}_{\mathrm{ss}}$ should be the same for b.i.d. and q.d. regimens for the same total daily dose. Subjects with moderate renal impairment are expected to have a $43 \%$ increase in apixaban exposure; however, apixaban's therapeutic utility index suggests that dose adjustment is not needed in this population. From Leil et al. [53] 
natural role of CTS in this field, so far its use has been relatively limited. Very few examples exist in which personalisation of treatment has been based on clinical relevance, rather than on pure scientific rationale. Recently, Albers et al. used simulations to assess the implications of a new age-based dosing strategy for carvedilol. The study showed that higher doses in younger patients (with respect to body weight) are needed to achieve the same exposure as adults [50]. Likewise, a CTS has been used for diclofenac as the basis for the evaluation of an effective and safe dosing regimen for acute pain in children [51].

Albeit a constant theme in scientific and regulatory forums, the use of personalised medicine concepts in paediatric scenarios remains wishful thinking. Both the FDA and the European regulatory authorities are increasingly requesting risk-benefit analyses of medicines. However, such appeals are not accompanied by suggested methods to be used in these analyses [52]. Furthermore, it has not become clear to most stakeholders that empirical methods are not suitable for the evaluation of multiple risk and benefit criteria, in particular in the presence of potential uncertainty because of the incompleteness of the evidence. Moreover, experimental evidence does not allow accurate assessment of the trade-offs of the benefits against the risks.

It can be anticipated that empirical evaluation of so many interacting factors cannot be defended without serious ethical and scientific issues. M\&S techniques are critical enablers for the implementation of personalised medicines and quantitative assessment of the risk-benefit ratio at individual and patient population levels. The use of a therapeutic utility index (TUI) illustrates such an endeavour. The concept has been introduced to enable the assessment of safety/efficacy of a treatment as a function of exposure. Using a model-based approach, Leil et al. show that renal impairment has no impact on efficacy/safety, despite significant differences in drug exposure [53].

\section{Conclusions}

The recent changes in the legislation regarding paediatric indications and the increasing understanding of the mechanisms and pathophysiology of paediatric diseases have created an unprecedented demand for evidence of the therapeutic benefit of new treatments in children. Such evidence cannot continue to be generated by empirical methods. There are simply not enough patients around to support drug development and approval processes in the same way as they are currently handled for adult indications. Moreover, even if availability of patients were not an issue, practical and ethical aspects cannot be overlooked.
Modelling and simulation can be used as a research tool to provide answers regarding the efficacy and safety of new drugs, in particular for paediatric and rare diseases. Despite some technical challenges, its potential value in paediatric research is indisputable and becomes greater as more data are accumulated throughout the development program. From a clinical and regulatory perspective, optimal use of M\&S may lead to fewer study failures and a smaller number of studies needed for generating the evidence required for the purposes of registration. As indicated previously, regulatory authorities have turned their interest towards the application of M\&S. However, to achieve the appropriate use of medicines in children (reducing the burden of clinical trials and improving efficacy and safety of therapeutic treatments in this population) guidelines should be implemented to recommend the proper use of M\&S techniques.

In conclusion, we have shown that $M \& S$ are valuable tools for integrating and quantifying the interaction among drug, disease and trial design factors. Although such clear-cut results cannot be obtained by traditional research protocols, M\&S continue to play a small, supportive role in the design of empirical clinical trials. It can be anticipated that, in the future, model-based approaches will become both the instrument and the aim of drug development programs, yielding quantitative evidence of the risk-benefit ratio for a given population or dosing regimen without the burden of trial and error.

Open Access This article is distributed under the terms of the Creative Commons Attribution Noncommercial License which permits any noncommercial use, distribution, and reproduction in any medium, provided the original author(s) and source are credited.

\section{References}

1. Lalonde RL, Kowalski KG, Hutmacher MM, Ewy W, Nichols DJ, Milligan PA, Corrigan BW, Lockwood PA, Marshall SA, Benincosa LJ, Tensfeldt TG, Parivar K, Amantea M, Glue P, Koide H, Miller R (2007) Model-based drug development. Clin Pharmacol Ther 82 (1):21-32

2. http://www.systemsbiology.org/. 2009

3. Danhof M, de Lange EC, Della Pasqua OE, Ploeger BA, Voskuyl RA (2008) Mechanism-based pharmacokinetic-pharmacodynamic (PK-PD) modeling in translational drug research. Trends Pharmacol Sci 29(4):186-191

4. Bakker BM, van Eunen K, Jeneson JA, van Riel NA, Bruggeman FJ, Teusink B (2010) Systems biology from micro-organisms to human metabolic diseases: the role of detailed kinetic models. Biochem Soc Trans 38(5):1294-1301

5. Musante CJ, Lewis AK, Hall K (2002) Small- and large-scale biosimulation applied to drug discovery and development. Drug Discov Today 7(20 Suppl):S192-S196

6. Michelson S (2006) The impact of systems biology and biosimulation on drug discovery and development. Mol Biosyst 2:288-291 
7. Manolis E, Pons G (2009) Proposals for model-based paediatric medicinal development within the current European Union regulatory framework. Br J Clin Pharmacol 68:493-501

8. Bruggeman FJ, Westerhoff HV (2007) The nature of systems biology. Trends Microbiol 15:45-50

9. Bangs A (2005) Predictive biosimulation and virtual patients in pharmaceutical R and D. Stud Health Technol Inform 111:3742

10. Cella M, de Vries Gorter F, Burger D, Danhof M, Della Pasqua O (2010) A model-based approach to dose selection in early pediatric development. Clin Pharmacol Ther 87(3):294 302

11. Abernethy DR, Burckart GJ (2010) Pediatric dose selection. Clin Pharmacol Ther 87(3):270-271

12. http://www.emea.europa.eu/htms/human/paediatrics/workshops. htm. 2009

13. Rodriguez W, Selen A, Avant D, Chaurasia C, Crescenzi T, Gieser G, Di Giacinto J, Huang SM, Lee P, Mathis L, Murphy D, Murphy S, Roberts R, Sachs HC, Suarez S, Tandon V, Uppoor RS (2008) Improving pediatric dosing through pediatric initiatives: what we have learned. Pediatrics 121(3):530-539

14. Ince I, de Wildt SN, Tibboel D, Danhof M, Knibbe CA (2009) Tailor-made drug treatment for children: creation of an infrastructure for data-sharing and population PK-PD modeling. Drug Discov Today 14:316-320

15. Anderson BJ, Allegaert K, Holford NH (2006) Population clinical pharmacology of children: general principles. Eur J Pediatr 165:741-746

16. Bouzom F, Walther B (2008) Pharmacokinetic predictions in children by using the physiologically based pharmacokinetic modelling. Fundam Clin Pharmacol 22:579-587

17. De Wildt SN, Knibbe CA (2009) Knowledge of developmental pharmacology and modeling approaches should be used to avoid useless trials in children. Eur J Clin Pharmacol 65:849-850

18. Cella M, Knibbe C, Danhof M, Della Pasqua O (2010) What is the right dose for children? Br J Clin Pharmacol 70(4):597603

19. Slikker W Jr, Young JF, Corley RA, Dorman DC, Conolly RB, Knudsen TB, Erstad BL, Luecke RH, Faustman EM, Timchalk C, Mattison DR (2005) Improving predictive modeling in pediatric drug development: pharmacokinetics, pharmacodynamics, and mechanistic modeling. Ann $\mathrm{N}$ Y Acad Sci 1053:505-518

20. ICH topic E11: clinical investigation of medicinal products in the paediatric population. 2001

21. Johnson TN (2005) Modelling approaches to dose estimation in children. Br J Clin Pharmacol 59:663-669

22. Rooney KF, Snoeck E, Watson PH (2001) Modelling and simulation in clinical drug development. Drug Discov Today 6:802-806

23. Duan JZ (2007) Applications of population pharmacokinetics in current drug labelling. J Clin Pharm Ther 32:57-79

24. Laer S, Barrett JS, Meibohm B (2009) The in silico child: using simulation to guide pediatric drug development and manage pediatric pharmacotherapy. J Clin Pharmacol 49:889904

25. Jumbe N, Yao B, Rovetti R, Rossi G, Heatherington AC (2002) Clinical Trial Simulation of a $200-\mu \mathrm{g}$ Fixed Dose of Darbepoetin Alfa in Chemotherapy-Induced Anemia. Oncology 16(Suppl 11): $37-44$

26. Laveille C, Vis $P$ (2008) Minutes from the round table held in Paris on July 6, 2007: 'contribution of modelling in the paediatric drug development'. Fundam Clin Pharmacol 22:609612

27. Rajman I (2008) PK/PD modelling and simulations: utility in drug development. Drug Discov Today 13:341-346
28. Theil FP, Guentert TW, Haddad S, Poulin P (2003) Utility of physiologically based pharmacokinetic models to drug development and rational drug discovery candidate selection. Toxicol Lett 138:29-49

29. Van de Waterbeemd H, Gifford E (2003) ADMET in silico modelling: towards prediction paradise. Nat Rev Drug Discov 2:192-204

30. Jones HM, Gardner IB, Watson KJ (2009) Modelling and PBPK simulation in drug discovery. AAPS J 11:155-166

31. Huntjens DR, Danhof M, Della Pasqua OE (2005) Pharmacokineticpharmacodynamic correlations and biomarkers in the development of COX-2 inhibitors. Rheumatology Oxford 44:846-859

32. Brent RL (2004) Utilization of juvenile animal studies to determine the human effects and risks of environmental toxicants during postnatal developmental stages. Birth Defects Res B Dev Reprod Toxicol 71:303-320

33. http://www.simcyp.com/. 2009

34. Chatelut E (2008) Population approaches in paediatrics. Fundam Clin Pharmacol 22:575-578

35. Yim DS, Zhou H, Buckwalter M, Nestorov I, Peck CC, Lee H (2005) Population pharmacokinetic analysis and simulation of the time-concentration profile of etanercept in pediatric patients with juvenile rheumatoid arthritis. J Clin Pharmacol 45(3):246256

36. Knibbe CA, Melenhorst-de Jong G, Mestrom M, Rademaker CM, Reijnvaan AF, Zuideveld KP, Kuks PF, van Vught H, Danhof M (2002) Pharmacokinetics and effects of propofol 6\% for shortterm sedation in paediatric patients following cardiac surgery. $\mathrm{Br} \mathrm{J}$ Clin Pharmacol 54:415-422

37. Ihmsen H, Viethen V, Forst J, Schwilden H, Schmitt HJ, Muenster $T$ (2009) Pharmacodynamic modelling of rocuronium in adolescents with Duchenne muscular dystrophy. Eur J Anaesthesiol 26:105-110

38. Tod M (2008) Evaluation of drugs in pediatrics using K-PD models: perspectives. Fundam Clin Pharmacol 22:589-594

39. Aarons L, Karlsson MO, Mentre F, Rombout F, Steimer JL, van Peer A (2001) Role of modelling and simulation in phase I drug development. Eur J Pharm Sci 13:115-122

40. Colburn WA (2003) Biomarkers in drug discovery and development: from target identification through drug marketing. J Clin Pharmacol 43:329-341

41. Krudys KM, Greenbaum CJ, Pihoker C, Vicini P (2008) Use of oral glucose minimal model-derived index of insulin sensitivity in subjects with early type 1 diabetes mellitus. Metabolism 57 (4):445-447

42. Breda E, Cavaghan MK, Toffolo G, Polonsky KS, Cobelli C (2001) Oral glucose tolerance test minimal model indexes of beta-cell function and insulin sensitivity. Diabetes 50(1):150 158

43. Santen G, van Zwet E, Danhof M, Della Pasqua O (2009) From trial and error to trial simulation. I. The importance of modelbased drug development for antidepressant drugs. Clin Pharmacol Ther 86(3):248-254

44. Gobburu JVS, Lesko LJ (2009) Quantitative Disease, Drug and Trial Models. Annu Rev Pharmacol Toxicol 49: 291-301

45. Girard P (2005) Clinical trial simulation: a tool for understanding study failures and preventing them. Basic Clin Pharmacol Toxicol 96:228-234

46. Onar A, Kocak M, Boyett JM (2009) Continual reassessment method vs. traditional empirically based design: modifications motivated by phase I trials in pediatric oncology by the Pediatric Brain Tumor Consortium. J Biopharm Stat 19(3):437455

47. Nguyen L (2008) Integration of modelling and simulation into the development of intravenous busulfan in paediatrics: an industrial experience. Fundam Clin Pharmacol 22:599-604 
48. Laer S, Elshoff JP, Meibohm B, Weil J, Mir TS, Zhang W, Hulpke-Wette M (2005) Development of a safe and effective pediatric dosing regimen for sotalol based on population pharmacokinetics and pharmacodynamics in children with supraventricular tachycardia. J Am Coll Cardiol 46:1322-1330

49. Maas HJ, Danhof M, Della Pasqua OE (2009) Analysis of the relationship between age and treatment response in migraine. Cephalalgia 29(7):772-780

50. Albers S, Meibohm B, Mir TS, Laer S (2008) Population pharmacokinetics and dose simulation of carvedilol in paediatric patients with congestive heart failure. Br J Clin Pharmacol 65:511-522
51. Standing JF, Howard RF, Johnson A, Savage I, Wong IC (2008) Population pharmacokinetics of oral diclofenac for acute pain in children. Br J Clin Pharmacol 66:846-853

52. Mussen F, Salek S, Walker S (2007) A quantitative approach to benefit-risk assessment of medicines. I. The development of a new model using multi-criteria decision analysis. Pharmacoepidemiol Drug Saf 1 [16 Suppl]:S2-S15

53. Leil TA, Feng Y, Zhang L, Paccaly A, Mohan P, Pfister M (2010) Quantification of apixaban's therapeutic utility in prevention of venous thromboembolism: selection of phase III trial dose. Clin Pharmacol Ther 88(3):375-382 